\title{
Vector meson fragmentation using a model with broken $S U(3)$ at the Next-to-Leading Order
}

\author{
H. Saveetha ${ }^{1}$, D. Indumathi ${ }^{2}$, Subhadip Mitra ${ }^{3}$ \\ ${ }^{1}$ Department of Theoretical Physics, \\ University of Madras, Chennai 600 025, India. \\ ${ }^{2}$ Institute of Mathematical Sciences, Chennai 600 113, India. \\ ${ }^{3}$ Laboratoire de Physique Theorique d'Orsay, Univ. Paris-Sud 11, France.
}

(Dated: July 29, 2018)

\begin{abstract}
A detailed study of fragmentation of vector mesons at the next-to-leading order (NLO) is given for $e^{+} e^{-}$scattering. A model with broken $S U(3)$ symmetry uses three input fragmentation functions $\alpha\left(x, Q^{2}\right), \beta\left(x, Q^{2}\right)$ and $\gamma\left(x, Q^{2}\right)$ and a strangeness suppression parameter $\lambda$ to describe all the light quark fragmentation functions for the entire vector meson octet. At a starting low energy scale of $Q_{0}^{2}=1.5(\mathrm{GeV})^{2}$ for three light quarks $(u, d, s)$ along with initial parameterization, the fragmentation functions are evolved through DGLAP evolution equations at NLO and the crosssection is calculated. The heavy quarks contribution are added in appropriate thresholds during evolution. The results obtained are fitted at the momentum scale of $\sqrt{s}=91.2 \mathrm{GeV}$ for LEP and SLD data. Good-quality fits are obtained for $\rho, K^{*}, \omega$ and $\phi$ mesons, implying the consistency and efficiency of this model that explains the fragmentation functions of vector mesons both at the leading and the next to leading order in QCD.
\end{abstract}

Keywords: vector meson, fragmentation, SU(3) symmetry, NLO . 


\section{INTRODUCTION}

The fragmentation of partons (quarks, gluons) into the desirable hadrons is called hadronization or fragmentation and are expressed in terms of functions named fragmentation functions. Fragmentation process can be understood only through phenomenological studies as perturbative Quantum Chromodynamics (pQCD) itself cannot explain it in a direct way. Various phenomenological studies ([1], [2], [3], [4], [5]) are being done for many years to understand the fragmentation process $\left(e^{+} e^{-}, e p, p p\right.$, lepton-nuclues, ...) at the leading order (LO) level and the next to leading order(NLO) level for pions and kaons (pseudoscalar)as well as baryons.

However, while considering vector mesons there is no such work has been found so far due to the paucity of the data. This motivated us to study the fragmentation of vector mesons. A model with broken $S U(3)$ [6],[7] is used in order to explain these fragmentation functions at the leading order level intially for $e^{+} e^{-}$annihilation and $p p$, collision processes [8]. As a consequence, it is aimed to extend the analysis for these vector mesons at the next to leading order using the same model with broken $S U(3)$. The significance of studying these vector mesons at the next-to-leading order level, particularly for the $\phi$ meson, will be a guideline for sure to understand the nucleon-nucleon scattering in the RHIC for Quark Gluon Plasma (QGP) studies. This work presents the study of fragmentation functions for $e^{+} e^{-}$at NLO.

Section 2 of the paper explains the cross section details of $e^{+} e^{-}$scattering at the Next to leading order level. Section 3 describes the outline of the broken $S U(3)$ model. Section 4 gives the initial parameterization. Section 5 using the model presents the expressions of fragmentation functions of entire meson nonet. Section 6 analyses the results obtained by comparison with the data and discuss them in detail. Section 7 concludes the work.

\section{KINEMATICS}

\section{A. Hadron production at the Next to leading order level}

In this paper, the fragmentation of a parton into a colorless hadron is being studied for $e^{+} e^{-}$annihilation. The basic differential cross-section of this process has the following form $[9]$ :

$\frac{d \sigma_{e^{+} e^{-}}^{h}\left(x, y ; Q^{2}\right)}{d x d y}=N_{c} \frac{4 \pi \alpha_{\mathrm{e} . \mathrm{m} .}^{2}}{3 Q^{2}}\left[\frac{3}{2} f_{B, 1}^{h}\left(x, Q^{2}\right)-3 y(1-y) f_{B, 2}^{h}\left(x, Q^{2}\right)+\frac{3}{2}(1-2 y) f_{B, A}^{h}\left(x, Q^{2}\right)\right]$

where

$$
\begin{aligned}
Q^{2} & =\left(l_{1}+l_{2}\right)^{2}, \\
x & =\frac{2 h \cdot\left(l_{1}+l_{2}\right)}{Q^{2}}, \\
y & =\frac{h \cdot l_{1}}{h \cdot\left(l_{1}+l_{2}\right)} \\
& =\frac{1}{2}\left[1-\cos \theta_{\mathrm{CM}}\right], \\
B & =\text { gauge boson }(g, \text { Zor } W) .
\end{aligned}
$$

Here $x$ is the fraction of energy carried over by the hadron from its parent quark, where $x \equiv E_{\text {hadron }} / E_{\text {quark }}=\left(2 E_{h} / \sqrt{s}\right) \leq 1\left(\right.$ or $\left.x_{p} \equiv 2 p_{h} / \sqrt{s}\right)$ and $\mathrm{Q}=\sqrt{s}$ is the energy scale, 
where the analysis is done.Hence, after integrating over y $\left(f_{B, A}^{h}\right.$ term goes to zero), Eq. [1] with, for example, $Z$ boson as the propagator becomes

$$
\begin{aligned}
\frac{d \sigma_{e^{+} e^{-}}^{h}\left(x ; Q^{2}\right)}{d x} & =\left(\frac{N_{c} 4 \pi \alpha^{2}}{3 Q^{2}}\right)\left[\frac{3}{2} f_{Z, 1}^{h}\left(x, Q^{2}\right)-\frac{1}{2} f_{Z, 2}^{h}\left(x, Q^{2}\right)\right] \\
f_{Z, r}^{h}\left(x, Q^{2}\right) & =\sum_{P} \mathcal{C}_{r}^{P}\left(x, Q^{2}\right) \otimes \lambda_{Z}^{P} D_{P}^{h}\left(x, Q^{2}\right), r=1,2 \\
\lambda_{Z}^{F} & =\lambda_{Z}^{q_{f}}=\lambda_{Z}^{\bar{q}_{f}}=c_{q_{f}}, \\
\lambda_{Z}^{G} & =\sum_{f} \lambda_{Z}^{F}
\end{aligned}
$$

where $D_{p}^{h}\left(x, Q^{2}\right)$ is the fragmentation function for a parton $(p)$ that fragments into one of the hadrons $(h)$ which we are interested in. The expressions for charge factors $c_{q_{f}}$ of each quark of flavor $f$ in terms of electromagnetic charge $e_{f}$, vector and axial vector electroweak couplings are given in [8]. The coefficient functions, $\mathcal{C}_{r}^{P}\left(x, Q^{2}\right)$, are expressed in series of $\alpha_{s}\left(Q^{2}\right)$ :

$$
\begin{aligned}
& \mathcal{C}_{r}^{F}\left(x, Q^{2}\right)=\delta(1-x)+\frac{\alpha_{s}\left(Q^{2}\right)}{2 \pi} \mathcal{C}_{r}^{F(1)}(x)+\ldots \\
& \mathcal{C}_{r}^{G}\left(x, Q^{2}\right)=\frac{\alpha_{s}\left(Q^{2}\right)}{2 \pi} \mathcal{C}_{r}^{G(1)}(x)+\ldots
\end{aligned}
$$

where $\mathcal{C}_{r}^{P(i)}$ 's are now independent of $Q^{2}$. Hence at NLO,

$$
\begin{aligned}
f_{B, r}^{h}\left(x, Q^{2}\right)= & \left(\delta(1-x)+\frac{\alpha_{s}\left(Q^{2}\right)}{2 \pi} \mathcal{C}_{r}^{F(1)}(x)\right) \otimes\left[\sum_{F} \lambda_{B}^{F}\left\{D_{q_{f}}^{h}\left(x, Q^{2}\right)+D_{\bar{q}_{f}}^{h}\left(x, Q^{2}\right)\right\}\right] \\
& +\frac{\alpha_{s}\left(Q^{2}\right)}{2 \pi} \mathcal{C}_{r}^{G(1)}(x) \otimes \lambda_{B}^{G} D_{G}^{h}\left(x, Q^{2}\right)
\end{aligned}
$$

From the above equation it is clear that at NLO, the presence of gluon term in the cross-sec equation contributes explicitly in the beginning itself at low $Q^{2}$ (for LO case it contributes only through evolution of $D_{q}$ 's). For $r=1,2$, the fragmentation functions (FFs) $D_{P}^{h}\left(x, Q^{2}\right)$, dependent on $Q^{2}$, are written explicitly at NLO as

$$
\begin{aligned}
f_{B, r}^{h}\left(x, Q^{2}\right)= & \sum_{F} \lambda_{B}^{F} \int_{x}^{1} \frac{d z}{z}\left(\delta(1-z)+\frac{\alpha_{s}\left(Q^{2}\right)}{2 \pi} \mathcal{C}_{r}^{F(1)}(z)\right)\left\{D_{q_{f}}^{h}\left(\frac{x}{z}, Q^{2}\right)+D_{\bar{q}_{f}}^{h}\left(\frac{x}{z}, Q^{2}\right)\right\} \\
& +\lambda_{B}^{G} \frac{\alpha_{s}\left(Q^{2}\right)}{2 \pi} \int_{x}^{1} \frac{d z}{z} \mathcal{C}_{r}^{G(1)}(z) D_{G}^{h}\left(\frac{x}{z}, Q^{2}\right) .
\end{aligned}
$$

Hence,

$$
\begin{aligned}
\frac{d \sigma_{e^{+} e^{-}}^{h}\left(x ; Q^{2}\right)}{d x} & =\left(\frac{N_{c} 4 \pi \alpha^{2}}{3 Q^{2}}\right)\left[\frac{3}{2} f_{B, 1}^{h}\left(x, Q^{2}\right)-\frac{1}{2} f_{B, 2}^{h}\left(x, Q^{2}\right)\right] \\
\frac{1}{\sigma_{t o t}}\left(\frac{d \sigma_{e^{+} e^{-}}^{h}\left(x ; Q^{2}\right)}{d x}\right) & =\frac{1}{\sum_{F} \lambda_{B}^{F}\left(1+\frac{\alpha_{s}}{\pi}\right)}\left[f_{B}^{h}\left(x, Q^{2}\right)\right]
\end{aligned}
$$


where,

$$
\begin{aligned}
f_{B}^{h}\left(x, Q^{2}\right)= & \int_{x}^{1} \frac{d z}{z}\left[\sum_{F} \lambda_{B}^{F}\left(\delta(1-z)+\frac{\alpha_{s}\left(Q^{2}\right)}{2 \pi} \mathcal{C}^{F(1)}(z)\right)\left\{D_{q_{f}}^{h}+D_{\bar{q}_{f}}^{h}\right\}\left(\frac{x}{z}\right)\right. \\
& \left.+\frac{\alpha_{s}\left(Q^{2}\right)}{2 \pi} \lambda_{B}^{G} \mathcal{C}^{G(1)}(z) D_{G}^{h}\left(\frac{x}{z}\right)\right], \\
\sigma_{\text {tot }}= & N_{c} \sum_{F} \lambda_{B}^{F}\left(\frac{4 \pi \alpha^{2}}{3 Q^{2}}\right)\left(1+\frac{\alpha_{s}}{\pi}\right), \text { at NLO. }
\end{aligned}
$$

Eq. (16) contains the complete set of equations for cross-section upto next-to-leading order level. The expressions for co-efficient functions $\mathcal{C}^{F(1)}(z)$ and $\mathcal{C}^{G(1)}(z)$ of Eq. (16) are taken from Appendix II of [9].

Perturbative Functions : The evolution of the parton fragmentation function $D_{i}\left(x, Q^{2}\right)$ with $Q^{2}$ is given by DGLAP evolution equations [11]:

$$
Q^{2} \frac{d}{d Q^{2}} D_{P}^{h}\left(x, Q^{2}\right)=\sum_{j} \mathcal{P}_{j i}\left(x, \alpha_{s}\right) \otimes D_{j}^{h}\left(x, Q^{2}\right)=\sum_{j} \int_{x}^{1} \frac{d z}{z} D_{j}^{h}\left(\frac{x}{z}, Q^{2}\right) \mathcal{P}_{j i}\left(z, \alpha_{s}\right),
$$

where $\mathcal{P}_{j i}$ 's are the time-like splitting functions and can be expressed as power series in $\alpha_{s}$ [10]:

$$
\mathcal{P}_{j i}\left(x, \alpha_{s}\right)=\frac{\alpha_{s}}{2 \pi} \mathcal{P}_{j i}^{(0)}(x)+\left(\frac{\alpha_{s}}{2 \pi}\right)^{2} \mathcal{P}_{j i}^{(1)}(x)+\ldots
$$

Expressions for $\alpha_{s}$ and time-like splitting functions to NLO are taken from Refs. [9],[12] and [13] where they were discussed in detail. The main difference between LO and NLO cross-sections are perturbative expansion of functions like $\alpha\left(x, Q^{2}\right)$ and time like splitting functions $P_{j i}$ which appears in the evolution equations. The expression for first term in Eq. (16) gives the LO term [10].

$$
\frac{1}{\sigma_{t o t}} \frac{\mathrm{d} \sigma^{h}}{\mathrm{~d} x}=\frac{\sum_{q} c_{q} D_{q}^{h}\left(x, Q^{2}\right)}{\sum_{q} c_{q}},
$$

where $D_{q}^{h}\left(x, Q^{2}\right)$ are analogously evolved to LO as well.

Eq. (19) can be written in terms of singlet and non-singlet combinations including the coefficient functions [8] having three light quarks $(u, d$ and $s)$ at the starting scale and evolved to the $Z$-pole with the heavy quarks $c$ and $b$ contributing during the evolution.

Vector mesons production of $\rho$ and $\omega$ s has been studied for LEP data [17],[19]. However, in the case of $K^{*}$ and $\phi$ instead of LEP [18],[20], SLD pure "uds" data (three flavors alone) [21] is used in order to avoid the contamination of heavy flavor mesons decay in the data as they decay preferably to one of these two strange mesons since $\left|V_{c s}\right|$ is large.

\section{MODEL FOR VECTOR MESON FRAGMENTATION}

This section gives the outline of the broken $S U(3)$ model that we will use in our analysis. Detailed explanations can be seen in our earlier work [8]. 
Perturbative QCD evolves these fragmentation functions through DGLAP evolution equations [11] to say, upto $Z$-pole, once they are defined by means of parameterization at a starting energy scale, $Q_{0}^{2}=1.5 \mathrm{GeV}^{2}$.

The fragmentation functions can be parameterized by comparison with the experimental data. A model is needed to determine these fragmentation functions and we use a model with broken $S U(3)$ [8]. In this paper, the fragmentation of the vector mesons $\rho\left(\rho^{+}, \rho^{-}, \rho^{0}\right)$, $K^{*}\left(K^{*+}, K^{*-}, K^{* 0}, \bar{K}^{* 0}\right), \omega$ and $\phi$ is studied. For each meson, six quark and anti-quark fragmentation functions, $D_{f}^{h}\left(x, Q^{2}\right), f=u, d, s, \bar{u}, \bar{d}$, overlines, and a gluon fragmentation function $D_{g}^{h}\left(x, Q^{2}\right.$ associated with the meson production, needs to be described at the starting scale.

Though $S U(3)$ flavor symmetry is not an exact symmetry, still it holds good in describing the octet of vector mesons with a symmetry breaking parameter. This model succeeded in explaining the octet mesons at LO in QCD with appreciable results for both $e^{+} e^{-}$and $p p$ scattering [8].

To explain the structure and purpose of the model let us begin with applying the $S U(3)$ symmetry for a general process:

$$
q_{i} \rightarrow h_{j}^{i}+X_{j}
$$

Under $S U(3)$ this will be written as $3 \rightarrow 8+X$ where 8 represents the octet hadron and $X$ are the debris that comes out along with hadron. This implies that a quark fragment into an octet hadron such that the possibilities for $X$ are either triplet $(3)$, antisixplet $(\overline{6})$ or fifteenplet (15). Let $\alpha\left(x, Q^{2}\right), \beta\left(x, Q^{2}\right)$ and $\gamma\left(x, Q^{2}\right)$ be the corresponding unknown $\operatorname{SU}(3)$ symmetric independent fragmentation functions for each of these possibilities [6], that is, for $\mathrm{X}$ to be 3 the probability of the quark to fragment into an octet hadron is $\alpha$. In a similar way, the other two $X(=\overline{6}, 15)$ values corresponds to the following fragmentation functions $\beta$ and $\gamma$.

Likewise, an anti-quark also produces an octet hadron with $X$ being an anti-triplet $(\overline{3})$, sixplet (6) or anti-fifteenplet $(\overline{15})$, for which $\bar{\alpha}\left(x, Q^{2}\right), \bar{\beta}\left(x, Q^{2}\right)$ and $\bar{\gamma}\left(x, Q^{2}\right)$ have to be determined.

To study the meson octet, we have a total of $56(8 \times 7)$ unknown fragmentation functions that has to be fitted with the data which is not an easier case to deal. Thus introducing this $S U(3)$ symmetry reduces the complexity of 56 unknown fragmentation functions into just seven fragmentation functions for all the mesons in the octet. This is further reduced by charge conjugation symmetry to just four, $\alpha, \beta, \gamma$ and the gluon, $D_{g}$.

\section{A. Representation of Fragmentation Functions}

All the octet mesons are represented by means of the above mentioned three independent fragmentation functions given in Table I. In addition to the symmetry property, isospin and charge conjugation invariance of vector mesons $\rho\left(\rho^{+}, \rho^{-}, \rho^{0}\right), K^{*}\left(K^{*+}, K^{*-}, K^{* 0}, \bar{K}^{* 0}\right)$ reduces the three independent unknown quark fragmentation functions further into functions named valence $(V)$ and sea $(\gamma)$. Assuming sea as flavor symmetric, the functions can be written as follows:

$$
\begin{gathered}
V\left(x, Q^{2}\right)=\alpha\left(x, Q^{2}\right)-\frac{3}{4} \gamma\left(x, Q^{2}\right), \\
S\left(x, Q^{2}\right)=2 \gamma\left(x, Q^{2}\right) .
\end{gathered}
$$


Eqs .(20) and (21) are the two well-defined expressions which describes the quark fragmentation function for all the mesons in the octet that are produced.

Breaking Parameter : Since $S U(3)$ is not a good description of octet, a $x$-independent parameter $\lambda$ is introduced in order to explain the strangeness suppression for $K^{*}$ meson. As the sea is flavor symmetric, suppression is common for all the light quarks either $u, d$ or $s$. Meanwhile, the $u$ and $d$ light quarks need suppression factor $\lambda$ in order to pick up a massive strange quark $s$ from sea. But in the case of strange quark it does not need such factor as it will easily pick up the other two quarks from the sea.

\section{INITIAL PARAMETERIZATION}

The inputs of valence $V\left(x, Q^{2}\right)$, sea $\gamma\left(x, Q^{2}\right)$ and gluon $D_{g}\left(x, Q^{2}\right)$ fragmentation functions at a starting scale of $Q_{0}^{2}=1.5 \mathrm{GeV}^{2}$ can be parameterized by means of a standard polynomial,

$$
F_{i}(x)=a_{i} x^{b_{i}}(1-x)^{c_{i}}\left(1+d_{i} x+e_{i} x^{2}\right),
$$

where $a_{i}, b_{i}, c_{i}, d_{i}$ and $e_{i}$ are the values to be determined. The contribution of heavy quark flavors are zero at the starting scale, but they are added in the appropriate thresholds during evolution.

\section{MESON FRAGMENTATION}

\section{A. Pure octet}

Combination of functions for $\rho$ and $K^{*}$ : The singlet combination of valence and sea fragmentation for one of the $\rho$ and $K^{*}$ s are given as (for more details, see Ref. [8]):

$$
\begin{gathered}
D_{0}^{\rho^{+}}=D_{u+\bar{u}+d+\bar{d}+s+\bar{s}}^{\rho^{+}}=2 V+12 \gamma \\
D_{0}^{K^{*+}}=D_{u+\bar{u}+d+\bar{d}+s+\bar{s}}^{K^{*+}}=(1+\lambda) V+12 \lambda \gamma .
\end{gathered}
$$

The term $\lambda$ in Eq .(24) refers to the strangeness suppression parameter. We can also write the non-singlet combinations in the same way. In addition to this, a suppression factor $f^{K^{*}}$ with $D_{g}^{K^{*}}=f_{g}^{K^{*}} D_{g}^{\rho}$ for gluons is introduced.

\section{B. Mixture of octet and singlet}

The broken $S U(3)$ model is now extended to $\omega$ and $\phi$ mesons which are orthogonal combinations of the $S U(3)$ octet $\left(\omega_{8}\right)$ and singlet states $\left(\omega_{1}\right)$.

$$
\begin{aligned}
\omega & =\sin \theta \omega_{8}+\cos \theta \omega_{1}, \\
\phi & =\cos \theta \omega_{8}-\sin \theta \omega_{1},
\end{aligned}
$$

where $\omega_{8}=(u \bar{u}+d \bar{d}-2 s \bar{s}) / \sqrt{6}, \omega_{1}=(u \bar{u}+d \bar{d}+s \bar{s}) / \sqrt{3}$ are the corresponding orthogonal states and $\theta$ is the vector mixing angle. 


\section{Singlet hadron $\left(\omega_{1}\right)$ fragmentation}

Consider the same process which is discussed earlier in the octet case.

$$
q_{i} \rightarrow h+X_{i}
$$

in which a quark hadronises into a singlet meson so that $X$ can only be a triplet $(3 \rightarrow 1+X)$. Therefore, we need to determine only one unknown fragmentation function $\delta\left(x, Q^{2}\right)$ in the singlet case. It is already known that the probability of a quark fragmenting into an hadron with $X$ being triplet is $\alpha\left(x, Q^{2}\right)$. Hence we use the simple ansatz that the function $\delta$ is simply related to $\alpha\left(x, Q^{2}\right)$, the fragmentation function for members of octet meson.

$$
\frac{\delta}{3}=\frac{f_{1} \alpha}{3}=\frac{f_{1}}{3}\left(V+\frac{3}{4} \gamma\right)
$$

where the factor $1 / 3$ is normalisation term and the proportionality constant parameter $f_{1}$ has to be determined in the analysis. Having both octet and singlet terms, the expressions for singlet and non-singlet combinations are presented further below.

\section{Combination of functions for $\omega$ and $\phi$}

a. Octet part : Let us begin with $\omega_{8}$ as it falls under pure $S U(3)$ octet. The fragmentation functions for light quarks $(u, d$ and $s)$ given in the Table I can be written as follows:

$$
\begin{aligned}
& D_{u}^{8}=\frac{V}{6}+2 f_{\text {sea }} \gamma ; \\
& D_{s}^{8}=\frac{2}{3} \lambda V+2 f_{\text {sea }} \gamma .
\end{aligned}
$$

The new parameter $f_{\text {sea }}$ is the unknown suppression factor for the $\mathrm{SU}(3)$-symmetric sea fragmentation functions; however the other terms follow the same definitions.

b. Singlet part : Using our ansatz for the singlet hadron, we have

$$
\begin{aligned}
D_{u}^{1}=D_{d}^{1} & =\frac{f_{1}^{u}}{3}\left(V+\frac{3}{4} f_{\text {sea }} \gamma\right), \\
D_{s}^{1} & =\frac{f_{1}^{s}}{3}\left(\lambda V+\frac{3}{4} f_{\text {sea }} \gamma\right) .
\end{aligned}
$$

The suppression factors for the $u, d$ - and $s$-type singlet fragmentation functions are also to be determined from this analysis. In general, the expression for the fragmentation functions with vetor mixing angle $\theta$ are

$$
\begin{aligned}
& D_{i}^{\phi}=\left(c_{i}^{\phi}\right)^{2}\left(\cos ^{2} \theta \frac{D_{i}^{8}}{\left(c_{i}^{8}\right)^{2}}+\sin ^{2} \theta \frac{D_{i}^{1}}{\left(c_{i}^{1}\right)^{2}}\right) \\
& D_{i}^{\omega}=\left(c_{i}^{\omega}\right)^{2}\left(\sin ^{2} \theta \frac{D_{i}^{8}}{\left(c_{i}^{8}\right)^{2}}+\cos ^{2} \theta \frac{D_{i}^{1}}{\left(c_{i}^{1}\right)^{2}}\right) .
\end{aligned}
$$

the term $i$ refers to the three light quarks $(u, d$ and $s)$. The co-efficient function values are taken from [8]. As usual, we again parameterize the gluon fragmentation functions as $D_{g}^{\omega, \phi}=f_{g}^{\omega, \phi} D_{g}^{\rho}$ and have to determine their values from comparison with data. 


\section{DATA ANALYSIS AND RESULTS}

We started with the initial values for the parameters of fragmentation functions and other scale independent functions that were used at the leading order level (see Table II and III) in [8]. All the NLO terms are defined in the appropriate places at the starting scale of $Q_{0}^{2}=1.5 \mathrm{GeV}^{2}$ for only three light quarks $(u, d, s)$, whereas the heavy quarks are kept zero initially. The broken $S U(3)$ model describes well in a simple manner about the three light quarks at this low input scale. However when $Q^{2}$ evolves upto the $Z$ - pole the heavy quark contributions are added in the appropriate thresholds such that they contribute during the evolution. The expression for splitting functions, co-efficient functions, and running coupling constant are defined in the NLO cross-section. The evolution code uses an $x$-space evolution algorithm that is fast and efficient.

Analysis is done with the LEP data[[17],[19]] at $Z$-pole $\left(Q^{2}\right)=(91.2)^{2} \mathrm{GeV}^{2}$ for $\rho$ and $\omega$ mesons. In the case of $K^{*}$ and $\phi$ instead of using LEP data [[18],[20]], SLD pure uds data (three flavors alone) [21] are being used. The purpose of using SLD data for strange mesons $K^{*}$ and $\phi$ is in order to avoid the contamination of heavy mesons, like $B$ and $D$, decaying into one of the strange mesons which will mix up with the direct fragmentation of a quark into $K^{*}$ and $\phi$. Whereas in the case of non-strange mesons $\rho$ and $\omega$ the $K$ meson decays into the least massive meson like $\pi$ rather than $\rho$ or $\omega$; hence contamination from heavy quarks in these channels is negligible.

\section{A. Analysis of data}

Now, with the usual definitions for valence $V\left(x, Q^{2}\right)$, sea $\gamma\left(x, Q^{)}\right.$and gluon $\mathrm{g}\left(x, Q^{2}\right)$ fragmentation functions, and with the help of Eqs. (23) and (24), we fitted the data to $\rho$ and $K^{*}$ for $x$ values ranging from $0.01-1$. In general, the large $x$ behaviour is explained by valence $V\left(x, Q^{2}\right)$ and small $x$ behaviour by sea $\gamma\left(x, Q^{2}\right)$. The intermediate $x$ part is governed by both sea and gluons which was not very well determined at LO in $e^{+} e^{-}$scattering [8]. Since there were no gluon terms in LO cross-section the idea about gluon behaviour is completely ill-determined at that point, whereas in NLO it is expected to get reasonable values of gluon related fragmentation functions as well as constant parameters in the polynomial.

In the analysis, the statistical and systematical error bars were added in quadrature. Since the data at specific $x$ values quite differ from that averaged over $x$ bin values, the cross-section is calculated by averaging over $x$ bins as per the data. We have used $x_{p}$ values throughout the analysis where $p$ refers to the momentum scale (that is, $x \equiv 2 E / \sqrt{s}$ while $x_{p} \equiv 2 p \sqrt{s}$.

The valence and sea fragmentation functions parameterization are first determined with only $\rho$ and $K^{*}$ because, in principle, the valence contribution can be clearly predicted by pure non-strange $\rho$ meson and the sea part (including suppression factor $\lambda$ by $\rho$ and the strange meson $K^{*}$. Hence, while determining these parameters the $x$-independent parameter $\lambda$ which explains the strangeness suppression was also included in the evolution. After this the parameterization for gluons was also included and the data for all nonet mesons was simultaneously fitted, keeping all parameters including those for valence and sea, free, and refitting all parameters together.

Fig. 1 shows the result of the fit to both $\rho^{+,-}$as well as $\rho^{0}$. Note that isospin symmetry implies $D_{q}^{\rho+}+D_{q}^{\rho-}=2 D_{q}^{\rho 0}$. Fig. 2 shows the distribution for SLD "pure uds" data [21] of $K^{* 0}+\bar{K}^{* 0}$.

The main observation here is that after the minimization process we get the value of 
$\lambda$, suppression parameter, as $\lambda=0.07 \pm 0.01$. This is roughly the same as the value of $\lambda=0.063 \pm 0.01$ at LO level obtained in Ref. [8]. So $\lambda$ lies in the range $0.05 \leq \lambda \geq 0.07$ with $1 \sigma$ error bar (see Table III). This result again is close to the value $\lambda=0.08$ for pseudoscalar mesons [7] which implies that the $x$ independent suppression parameter is completely spinindependent quantity. The sea suppression factor $f_{g}^{K *}$ for strange meson $K^{*}$ came out to be $f_{g}^{K *}=1$, indicating no suppression. The $\chi^{2}$ values for the fit to both $\rho$ and $K^{*}$ data is given in Table (IV).
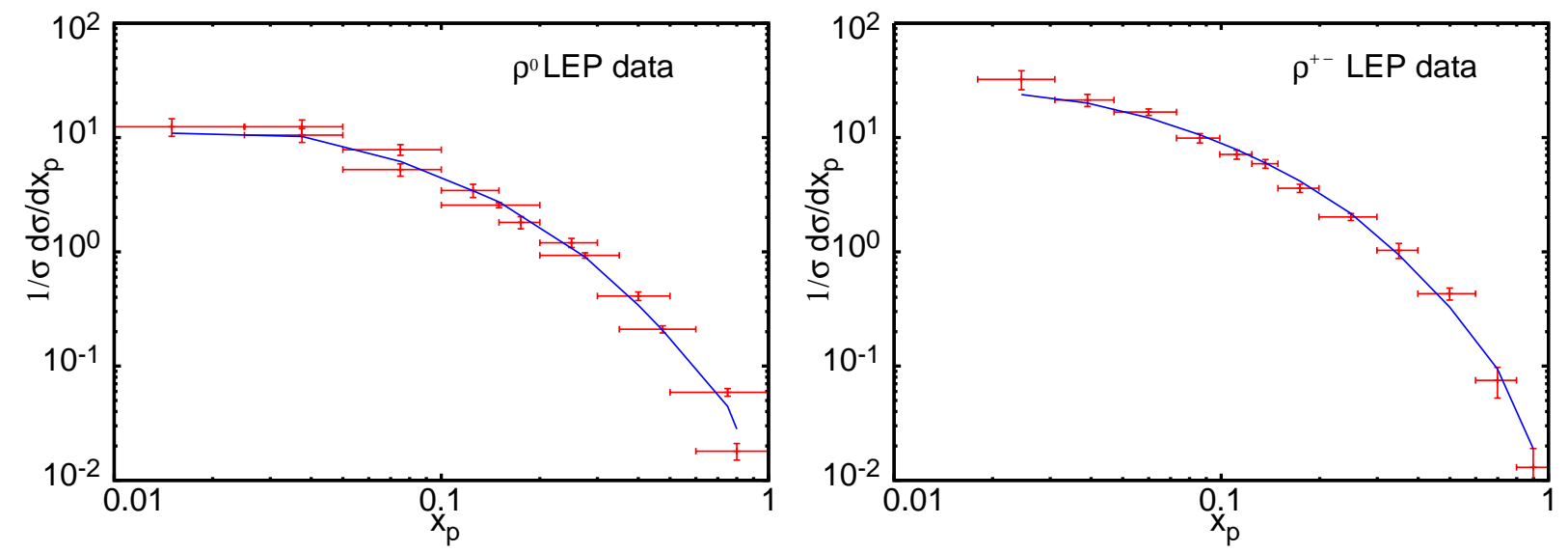

FIG. 1. Fit for rho meson in terms of fragmentation functions with (L) LEP data on the $Z$-pole for two data sets (ALEPH-thick line and DELPHI-thin line) of $\rho^{0}$ and (R) for $\rho^{+-}$[17]. The data are shown with statistical and systematic errors added in quadrature.

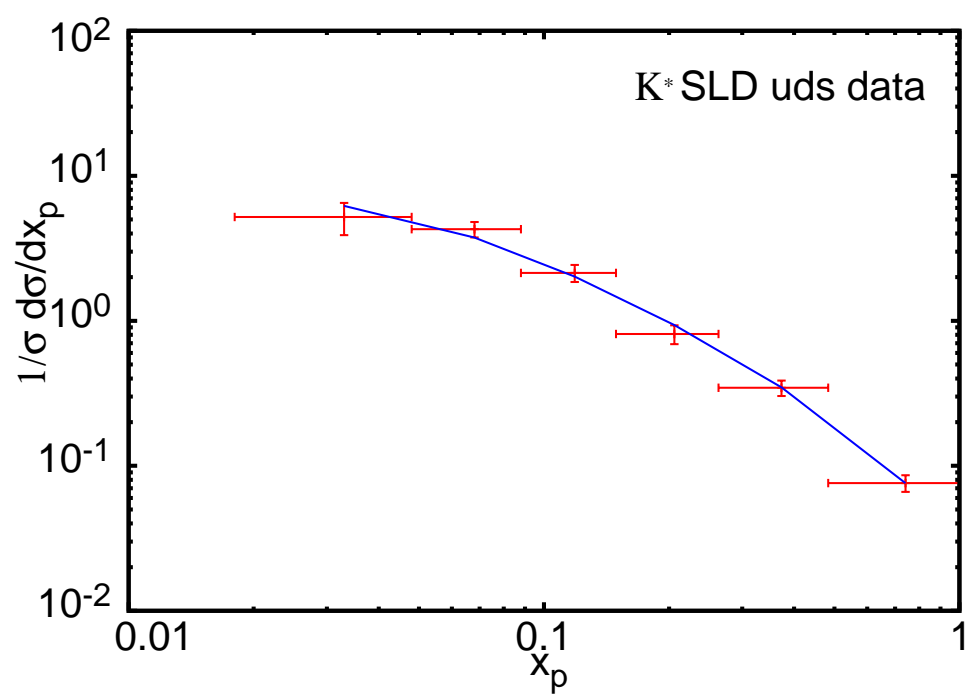

FIG. 2. Fit for $K^{*}$ meson with the best fit value of suppression factor $\lambda=0.07$. Data are taken from Ref. [21] at the $Z$-pole, from light quarks only and the smooth line refers the cross section at NLO.

In the case of $\omega$ and $\phi$ mesons, there are a few additional unknown parameters like $f_{1}^{u}, f_{1}^{s}$ and $f_{\text {sea }}$ for $\omega$ and $\phi$ which represents the singlet constants and sea suppression and mixing angle $\theta$ are to be determined. Since $\omega$ is highly dominated by $u$ and $d$ quarks it will behave as a purely non-strange meson, while $\phi$ is almost purely dominated by its $\sigma \bar{s}$ component. 

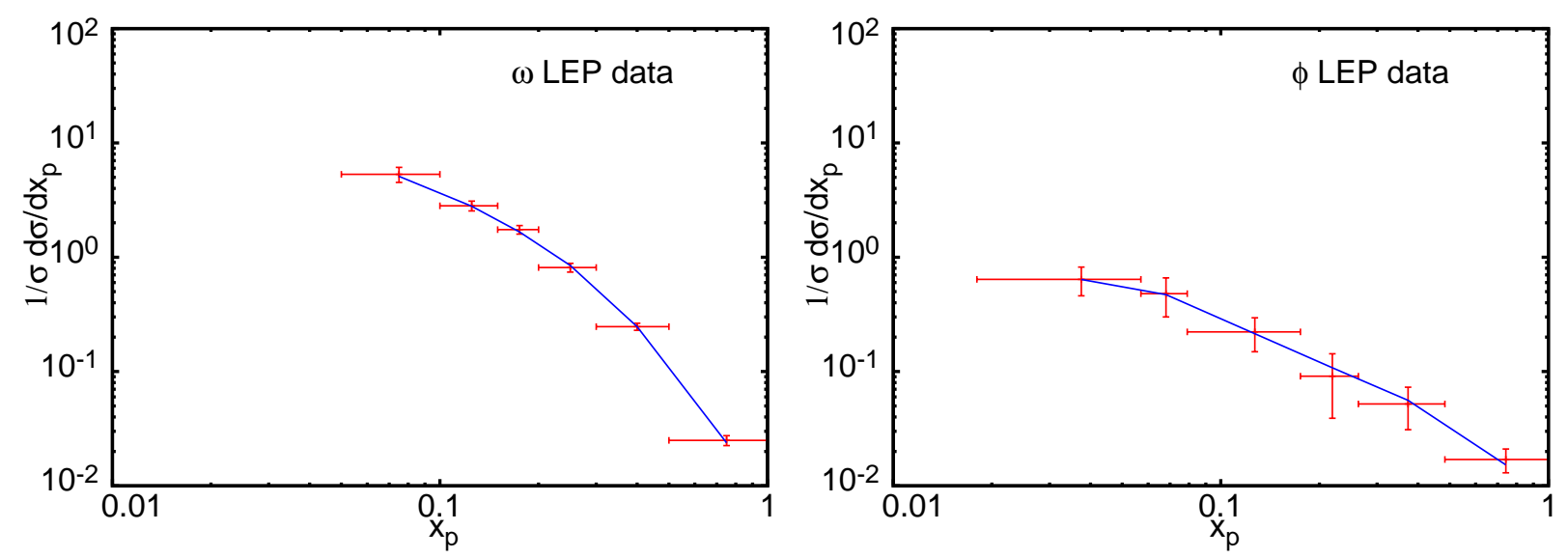

FIG. 3. Fits to omega (L) and (R) phi meson. The data correspond to LEP data for $\omega[19]$ and the SLD data from light quarks alone [21] for $\phi$ at NLO level.

The comparison of the best fit to the $\omega$ and $\phi$ data are shown in the Fig. 3. The $\phi$ meson, an interesting candidate for QGP studies, in the entire meson nonet, is also fitted to only light quark pure uds SLD data [20] as in the $K^{*}$ case.

The best-fit value of $\theta$, the vector mixing angle, is $\theta=39-42^{\circ}$ at $1 \sigma$. This value is reasonably close to the value of $\theta=42 \pm 2^{\circ}$ obtained in the earlier LO analysis [8]. This is also reasonably close to the value $\theta=36^{\circ}-38.7^{\circ}[14]$.

As discussed earlier in [8], the values of the constants $f_{1}^{s}$ of $\omega$ meson and $f_{1}^{u}$ of $\phi$ are kept zero. The other two constants are $f_{1}^{u}=0.05, f_{\text {sea }}^{\omega}=0.99$ which are still sensiblly close to the LO results and the constant factor for gluon suppression value $f_{g}^{\omega}=1$ with large errors.

The unknown constants $f_{\text {sea }}^{\phi}, f_{1}^{s}$ and $f_{g}^{\phi}$ of $\phi$ meson are also determined by fitting with the data. According to Ref. [15] the value of $\theta$ close to $35^{\circ}$ value saturates the physical $\phi$ state as a pure $s \bar{s}$ state. Thus we fixed the sea suppression factor for $\phi$ as $f_{\text {sea }}^{\phi}=\lambda^{2}$ as it contains dominantly strangeness $(s \bar{s})$ state, while $f_{1}^{s}=5.63$, again with larger errors.

The gluon suppression parameter for $\phi$ is tightly constrained as $f_{g}^{\phi}=0.4 \pm 0.04$ in contrast to hardly any suppression required in $\omega$. Hence it is clearly understood that $\phi$ is a dominantly strange meson with both sea and gluon contributions suppressed.

The best-fit values for all the parameters and their $1 \sigma$ errors are given in Table (III. It can be seen that all the polynomial constants in the three fragmentation functions are consistent with zero. The $\chi^{2}$ values of the individual fits to the different meson data is given in Table IV.

The global $\chi^{2}$ for entire meson nonet was 17.1 with 44 data points and 21 degrees of freedom. This reflects 70 percent confidence level for the goodness-of-fit which looks reasonable.

Hence with the broken $S U(3)$ model we are able to explain the entire meson octet with the introduction of very few fragmentation fuctions basically and some additional $x$ - and $Q^{2}$-independent parameters for extension of the octet and singlet mixture at the next-toleading order level. This proves the efficiency of the model to explain the sparse data with minimum number of fragmentation functions.

\section{DISCUSSION AND CONCLUSION}

Fragmentation functions for the vector meson nonet $\left(\rho\left(\rho^{+}, \rho^{-}, \rho^{0}\right), K^{*}\left(K^{*+}, K^{*-}, K^{* 0}, \bar{K}^{* 0}\right)\right.$, $\omega$ and $\phi)$ are studied for the first time at the next-to-leading order level for $e^{+} e^{-}$scattering. 
To achieve this we used a simple model named broken $S U(3)$, since $S U(3)$ is a fair description of octet mesons.

The model uses $S U(3)$ symmetry along with charge conjugation and isospin symmetry to describe the three input fragmentation functions $\alpha\left(x, Q^{2}\right), \beta\left(x, Q^{2}\right)$ and $\gamma\left(x, Q^{2}\right)$ (see Table (II)) at an input scale of $Q_{0}^{2}=1.5(\mathrm{GeV})^{2}$ for three light quarks $(u, d, s)$ where the heavy flavors like charm and bottom quarks were zero at this scale. These, along with the gluon fragmentation functions, were evolved to the $Q^{2}$ of the data. The heavy quark contributions were added in appropriate thresholds during the evolution.

With the standard input parameterization, these fragmentation functions were evolved through DGLAP evolution equations at NLO and the cross-sections were calculated for $\rho, K^{*}$ $\omega$ and $\phi$ mesons. The symmetry was broken when we introduced a $x$ independent parameter $\lambda$ for strange mesons to explain the strangeness suppression for non-strange mesons to pick up a strange quark from sea.

The results obtained were fitted with the momentum scale of $\sqrt{s}=91.2 \mathrm{GeV}$ for LEP $(\rho$ and $\omega)$ and SLD data $\left(K^{*}\right.$ and $\left.\phi\right)$ at the NLO level and the fit looks reasonable at NLO. The best fit values of the parameters for quark and gluon fragmentation functions $(V, \gamma, g)$ with the error bars were given in Table (II). The suppression parameter, $\lambda=0.07$ value obtained in NLO is almost equal to the LO result and still close to pseudoscalar meson $\lambda=0.08$ value. This clearly shows that $\lambda$ is a spin independent factor.

The values of the gluon fragmentation functions were well determined in this study. The behaviour both at small $x$ ( $x^{b}$ dependence) and at large $x\left((1-x)^{c}\right.$ dependence) in gluon fragmentation function lies between Valence and Sea fragmentation functions. That is at large $x$ the $\gamma\left(x, Q^{2}\right)$ falls first, then the gluon $g\left(x, Q^{2}\right)$, and finally $V\left(x, Q^{2}\right)$ towards the largest $x$ values. At LO, the gluon fragmentation appears only in the DGLAP evolution equation. The NLO cross sections, in contrast to the LO case, are directly dependent on the gluon fragmentation function. This is reflected in the tight constraints on the gluon fragmentation function parameters at NLO as seen in Table II. In particular, the fits are very sensitive to the small- $x$ behaviour of the gluon, which transforms from a very converging, vanishing function at low $Q^{2}$ to a highly diverging one at larger $Q^{2}$ due to the poles at $x=0$ in the relevant splitting functions. Specifically, it can be seen that the exponent $b$ in $x^{b}$ is extremely well-determined for the gluon fragmentation function.

In addition, it is seen that the gluon fragmentation functions are severely suppressed in $\phi$ but not in $\omega$, which are mixtures of $\mathrm{SU}(3)$ octet and singlet mesons. This is also reflected in the value of the singlet-octet mixing parameter $\theta$, close to $\theta \sim 41^{\circ}$. The values of the constants simply implies that $\omega$ is dominantly a non-strange meson and $\phi$ is dominantly a strange meson.

All the parameter values of quark and gluon fragmentation functions with the additional constant parameters with error bars were given in Tables (II) and (III). Finally the $\chi^{2}$ values for each meson were tabulated Table (IV).

In summary, we got reasonable fits for all the mesons at the NLO level for $e^{+} e^{-}$scattering which indeed implies the consistency and efficiency of this model that explains the fragmentation functions of vector mesons both at the leading and the next to leading order in QCD. This work will be extended further to $p p$ collision in future to understand further the $\phi$ meson in particular which forms the baseline to understand the QGP.

c. Acknowledgements : HS thanks A S Vytheeswaran for constant encouragement and support and M V N Murthy for discussions. 
[1] L. Bourhis, M. Fontannaz, J.Ph. Guillet, M. Werlen, Eur. Phys. J. C 19, 89, 2001.

[2] Stefan Kretzer Acta Phys.Polon. B 36, 179, 2005, arXiv:hep-ph/0410219.

[3] M. Hirai, S. Kumano, T.-H. Nagai, K. Sudoh, Phys. Rev. D 75, 094009, 2007.

[4] Daniel de Florian, Rodolfo Sassot, Marco Stratmann, Phys. Rev. D 76, 074033, 2007.

[5] Manuel Epele, Romina Llubaroff, Rodolfo Sassot, Marco Stratmann, Phys. Rev. D. 86, 074028, 2012.

[6] D. Indumathi, H. S. Mani, A. Rastogi, Phys. Rev. D 58, 094014 (1998), arXiv: hep$\mathrm{ph} / 9802324 \mathrm{v} 1,1998$.

[7] D. Indumathi, B. Misra, arXiv:0901.0228v1, 2009.

[8] D. Indumathi, H. Saveetha, IJMPA 27, 19, 1250103 (2012).

[9] W. Furmanski, R. Petronzio, Z. Phys. C 11, 293 ,1982.

[10] C. Amsler et al., Phy. Lett. B 667, 1 (2008).

[11] G. Altarelli, G. Parisi, Nucl. Phy. B 126, 298 (1977).

[12] W. Furmanski, R. Petronzio, CERN-TH.2933 (1980); Phys. Lett. B 97, 437 (1980).

[13] R. K. Ellis, W. J. Stirling and B. R. Webber, QCD and Collider Physics, Cambridge University Press, Cambridge (1996).

[14] K. Nakamura et al. (Particle Data Group), J. Phys. G 37, 075021 (2010).

[15] W.-M. Yao et al., J. Phys. G 33, 1 (2006).

[16] A compilation of inclusive particle production data in $e^{+} e^{-}$process is taken from G. D. Lafferty, P. I. Reeves and M. R. Whalley, J. Nucl. Part. Phys. G 21, A1-A151 (1995). Individual data for $\rho, K^{*}, \omega$ and $\phi$ at $Z$ pole are listed separately.

[17] D. Buskulic et al., ALEPH Collab., Z. Phys. C 69, 379 (1996);

R. Barate et al., ALEPH Collab., Phys. Rep. 294, 1 (1998);

P. Abreu et al., DELPHI Collab., Z. Phys. C 65, 587 (1995) G. D. Lafferty et al., J.Phys.G.21, A1-A151 (1995) for the $\rho^{0}$ and $\rho^{+-}$data on the $Z$-pole and S. Abachi et al., HRS Collab., Phys. Rev. D 40, 706 (1989) for the low energy $\rho$ data.

[18] D. Buskulic et al., ALEPH Collab., Z. Phys. C 69, 379 (1995);

R. Barate et al., ALEPH Collab., Phys. Rep. 294, 1 (1998);

P. Abreu et al., DELPHI Collab., Phys. Lett. B 298, 236 (1993);

R. Akers et al., OPAL Collab., Z. Phys. C 68, 1 (1995) for the inclusive $K^{*}$ data. For the "uds" data, see Ref. [21] below.

[19] D. Buskulic et al., ALEPH Collab., Z. Phys. C 69, 379 (1996);

R. Barate et al., ALEPH Collab., Phys. Rep. 294, 1 (1996) for the $\omega$ data.

[20] D. Buskulic et al., ALEPH Collab., Z. Phys. C 69, 379 (1996);

R. Barate et al., ALEPH Collab., Phys. Rep. 294, 1 (1998);

R. Akers et al., OPAL Collab., Z. Phys. C 68, 1 (1995);

P. Abreu et al., DELPHI Collab., Z. Phys. C 73, 61 (1996) for the inclusive $\phi$ data. For the "uds" data, see Ref. [21] below.

[21] K. Abe et al., Phys. Rev. D 59, 052001 (1999); K. Abe et al., ibid. 69, 072003 (2003). 


\begin{tabular}{|c|c|c|c|}
\hline $\begin{array}{c}\text { fragmenting } \\
\text { quark }\end{array}$ & $K^{*+}$ & $\begin{array}{c}\text { fragmenting } \\
\text { quark }\end{array}$ & $K^{* 0}$ \\
\hline$u$ & $: \alpha+\beta+\frac{3}{4} \gamma$ & $u$ & $: 2 \beta+\gamma$ \\
\hline$d$ & $: 2 \beta+\gamma$ & $d$ & $: \alpha+\beta+\frac{3}{4} \gamma$ \\
\hline$s$ & $: 2 \gamma$ & $s$ & $: 2 \gamma$ \\
\hline $\begin{array}{c}\text { fragmenting } \\
\text { quark }\end{array}$ & $\omega / \phi$ & $\begin{array}{c}\text { fragmenting } \\
\text { quark }\end{array}$ & $\rho^{0}$ \\
\hline$u$ & $: \frac{1}{6} \alpha+\frac{9}{6} \beta+\frac{9}{8} \gamma$ & $u$ & $: \frac{1}{2} \alpha+\frac{1}{2} \beta+\frac{11}{8} \gamma$ \\
\hline$d$ & $: \frac{1}{6} \alpha+\frac{9}{6} \beta+\frac{9}{8} \gamma$ & $d$ & $: \frac{1}{2} \alpha+\frac{1}{2} \beta+\frac{11}{8} \gamma$ \\
\hline$s$ & $: \frac{4}{6} \alpha+\frac{9}{6} \gamma$ & $s$ & $: 2 \beta+\gamma$ \\
\hline $\begin{array}{c}\text { fragmenting } \\
\text { quark }\end{array}$ & $\rho^{+}$ & $\begin{array}{c}\text { fragmenting } \\
\text { quark }\end{array}$ & $\rho^{-}$ \\
\hline$u$ & $\alpha \alpha+\beta+\frac{3}{4} \gamma$ & $u$ & $: 2 \gamma$ \\
\hline$d$ & $: 2 \gamma$ & $d$ & $: \alpha+\beta+\frac{3}{4} \gamma$ \\
\hline$s$ & $: 2 \beta+\gamma$ & $s$ & $: 2 \beta+\gamma$ \\
\hline $\begin{array}{c}\text { fragmenting } \\
\text { quark }\end{array}$ & $\overline{K^{* 0}}$ & $\begin{array}{c}\text { fragmenting } \\
\text { quark }\end{array}$ & $K^{*-}$ \\
\hline$u$ & $: 2 \beta+\gamma$ & $u$ & $: 2 \gamma$ \\
\hline$d$ & $: 2 \gamma$ & $d$ & $: 2 \beta+\gamma$ \\
\hline$s$ & $: \alpha+\beta+\frac{3}{4} \gamma$ & $s$ & $: \alpha+\beta+\frac{3}{4} \gamma$ \\
\hline
\end{tabular}

TABLE I. Quark fragmentation functions into members of meson octet in terms of the $\mathrm{SU}(3)$ functions, $\alpha, \beta$ and $\gamma$.

\begin{tabular}{|c|c|c|c|}
\hline & & Central Value & Error Bars \\
\hline$V$ & $a$ & 0.72 & 0.09 \\
\hline & $b$ & 0.52 & 0.15 \\
\hline & $c$ & 1.24 & 0.10 \\
\hline & $d$ & 0.27 & 0.28 \\
\hline & $e$ & -0.16 & 0.14 \\
\hline$\gamma$ & $a$ & 0.99 & 0.01 \\
\hline & $b$ & -0.48 & 0.02 \\
\hline & $c$ & 5.48 & 0.14 \\
\hline & $d$ & -0.09 & 0.18 \\
\hline & $e$ & 1.25 & 0.63 \\
\hline$D_{g}$ & $a$ & 3.89 & 0.41 \\
\hline & $b$ & 0.745 & 0.001 \\
\hline & $c$ & 3.14 & 0.19 \\
\hline & $d$ & -0.13 & 0.23 \\
\hline & $e$ & -0.21 & 0.44 \\
\hline
\end{tabular}

TABLE II. Best fit values of the parameters for the input fragmentation functions at the initial scale of $Q^{2}=1.5 \mathrm{GeV}^{2}$. 


\begin{tabular}{|c|c|c|}
\hline & Central Value & Error Bars \\
\hline$\lambda$ & 0.07 & 0.01 \\
\hline$\theta$ & 40.49 & 1.31 \\
\hline$f_{\text {sea }}^{\omega}$ & 0.99 & 0.08 \\
\hline$f_{1}^{u}(\omega)$ & 0.05 & 0.36 \\
\hline$f_{1}^{s}(\phi)$ & 5.63 & 2.16 \\
\hline$f_{g}^{K^{*}}$ & 1.00 & 0.09 \\
\hline$f_{g}^{\omega}$ & 1.00 & 0.38 \\
\hline$f_{g}^{\phi}$ & 0.40 & 0.04 \\
\hline
\end{tabular}

TABLE III. Best fit values of the parameters for the input fragmentation functions at the initial scale of $Q^{2}=1.5 \mathrm{GeV}^{2}$.

\begin{tabular}{|c|c|c|}
\hline Data Set & No. of data points & $\chi^{2}$ \\
\hline$\rho^{0}($ ALEPH $)$ & 8 & 5.6 \\
\hline$\rho^{0}$ (DELPHI 95$)$ & 6 & 2.4 \\
\hline$\rho^{+-}($OPAL $)$ & 12 & 6.1 \\
\hline$K^{* 0}($ SLD $)$ & 6 & 2.9 \\
\hline$\omega($ ALEPH $)$ & 6 & 0.3 \\
\hline$\phi($ SLD $)$ & 6 & 0.2 \\
\hline
\end{tabular}

TABLE IV $\chi^{2}$ for fits to inclusive vector meson production data on the $Z$-pole from LEP and SLD experiments with a total of 44 data points and 23 free parameters, we have a total $\chi^{2}=17.1$ for $44-23=21$ degrees of freedom. 